\title{
CONVERGING MULTIDOMAIN SERVICES IN AN IMS ENVIRONMENT FOR IPTV AND 3G NETWORKS
}

\author{
Sergio Gonzalez-Miranda ${ }^{1}$ Tomas Robles ${ }^{1}$ Fernando Ortigosa ${ }^{2}$ Juan Carlos \\ Plaza $^{2}$ and Jose Luis Peña ${ }^{3}$ \\ ${ }^{1}$ Technical University of Madrid (UPM), Madrid, Spain \\ \{miranda, trobles\} edit.upm.es \\ ${ }^{2}$ Ericsson Network Services, Madrid, Spain \\ \{ortigosa, plaza\} dericsson.com \\ ${ }^{3}$ Telefonica I+D, Madrid, Spain \\ penasedano@tid.es
}

\begin{abstract}
Convergent service environments are required to deal with current tendency to heterogeneity in network contents and its inherent incorporation of more types of devices demanding services from multiple domains. Efficient management architectures are required to tackle issues regarding: media content, subscriber reachability and subscriptions information coming from different types of networks in order to enable well organized communications among different environments such as: IMS, IPTv and subscribers management repositories.
\end{abstract}

This paper describes the work of Eureka Mobicome project on the definition of an architecture to manage and provide services among distinct service delivery platforms (IPTV and ENUM) using IMS as a common core and proposes a compound modular architecture to set the basis for Future Internet converging service provision. The proposed architecture has been validated by the Interconnection of different prototypes deployed at Madrid (Spain) and Oslo (Norway).

\section{KEYWORDS}

IMS, XDMS, IPTv, ENUM, Convergence, Future Internet, Services

\section{INTRODUCTION}

The Worldwide research activity focused on development activities of the original Internet, socalled Future Internet [1] is involved with several concepts such as: network infrastructure (virtualization, information), devices, interfaces and software. The resulting Service Delivery Platforms based on core networks require new network management mechanisms in order to achieve an optimal use of network resources that guarantees a reliable and robust orchestration of both access to/delivery of convergent services.

Mobicome is a Eureka project with partners from Spain, Sweden and Norway. The MOBICOME project [2] has as main goal the provision of unified user subscription management and service continuity for an IP Multimedia System (IMS) deployed on a fixed mobile convergent multi access environment. The project includes development of solutions that enable: 
- Seamless access to the different wireless technologies like UMTS, EDGE, Wireless LAN; etc.

- Unified identification, authentication and authorization of the users

- Unified service offering and provisioning in:

- Both mobile networks and fixed broadband networks

- Both on mobile terminals and stationary computers

- Service continuity when the user is roaming from mobile networks to fixed networks and vice versa.

Another goal is to enable the development of rich communication services which make use of all the communication channels such as voice, text, video, document, etc. in various ways depending on the context.

\section{SERVICE CONVERGENCE OVER IP NETWORKS}

As massive use and penetration of xDSL and UMTS grows, IPTV. VoIP and other multimedia and conversational services have been introduced into the telco service portfolio. Initially, they all were introduced as separate and independent services, each dealing with its own AAA and QoS samely separated. The same telecommunication equipments and controls were realized for every single service. This led to a service approach with multiplicity of functions.

As most control operations are shared for the service portfolio offered in a networked environment combining AAA, QoS and subscriber information storage and handling the IMS avoids this duplication by binding these functions in its control layer. This approach leads to PSTN, IPTv, VoIP, GSM networks convergence, aka Fixed Mobile Convergence (FMC) [3].

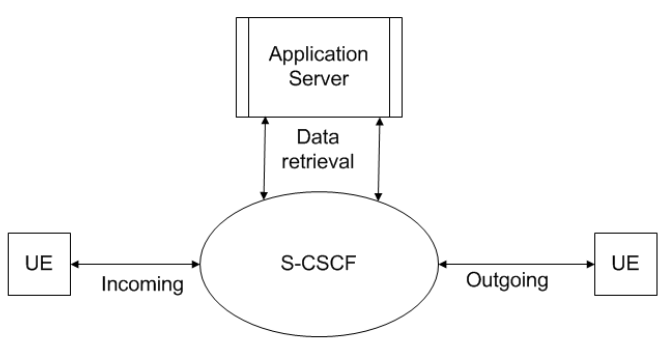

Figure 1. Service convergence components

In an effort to realize the vision of fixed mobile convergence, the telecom operators found very compelling to extend the usage of 3GPP IP Multimedia Subsystem, originally intended for the 3G mobile network to fixed broadband networks like xADSL, cable TV, IPTV, etc. However, there are many challenges that must be solved before the fixed mobile convergence vision based on IMS can be realized.

In fact, a mobile phone equipped with an IMS client will not be able to operate in a wireless LAN environment. It is not only because of the differences between IMS and the standard SIP (Session Initiation Protocol) but also because of the differences in terms of subscription fundament between the mobile world and the fixed one. Indeed, in the mobile world, each user is associated 
to one subscription while in the fixed network, a whole household with many users can share one subscription.

\section{SUPPORTING TECHNOLOGIES AND RELATED WORK}

All the relevant technologies involved in our research are described in this section, including: XDMS, IPTV and ENUM.

The elements orchestration allows users to make seamless use of all the available services in our IMS testbed.

\subsection{IMS}

The IP multimedia subsystem (IMS) as specified by the 3rd Generation Partnership Project (3GPP) [3] and the 3rd Generation Partnership Project 2 (3GPP2) is an architectural framework for delivering Internet Protocol (IP) multimedia services that can be adapted to support SIP (Session Initiation Protocol) -session-based services [4] [5].

The IP Multimedia Subsystem (IMS) [6] represents a suite of core network functional entities that enables a playground for the creation and deployment of current, new and future converging services.

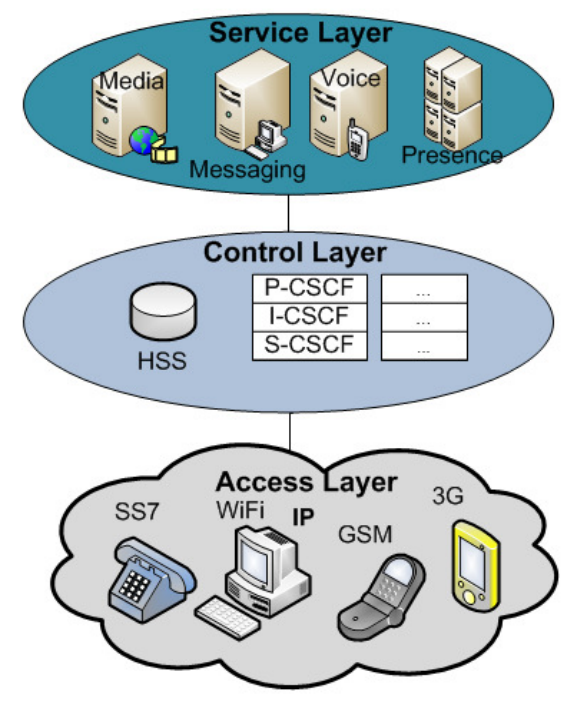

Figure 2. Service convergence over IMS control layer

Its access agnostic approach opens the service portfolio to multiple devices. Besides, IMS infrastructure provides great flexibility and adaptability to incorporate innovations in the modern communications ecosystem.

The application services support is done via the service layer relying on a number of network reference points defined to support operator-provided services.

The reference points defined in IMS also allow operation and interworking with a variety of external networks. 


\subsection{OMA XDM Service Enabler}

The Open Mobile Alliance (OMA) [7] is a standardization body dedicated to development of open standards for the mobile phone industry. Its members include both equipment and mobile systems manufacturer and mobile operators. Among its activities OMA has standardized several services enablers to allow interoperability across devices, service providers, operators, and networks. Among these service enablers, the XML Document Management Service (XDMS) [8] enables the handling of user-service related information via well structured XML documents. XCAP [9] is used as the common protocol to access and manipulate these XML documents which are stored in logical repositories in network (Fig. 3). These repositories are also referred to as XDMS or XML Document Management Servers.

Application Usage or Application Unique IDs (AUID) are used by applications to use a given document via XCAP.

The XDM enabler considers the reutilization of Application Usages by multiple service enablers.

Besides of the Presence Rules which supports the Presence Rules document storage in the XDMS and document subscription and notification management functions. Some other services mold a complete suite of enablers such as: Shared Lists, Shared Profiles, Shared Policies and Shared Groups.

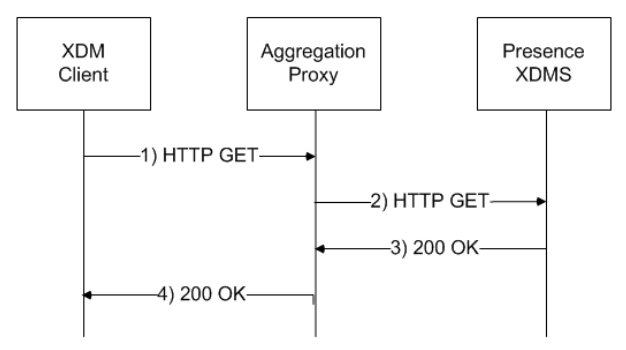

Figure 3. XDMS data flow

\subsection{IPTV}

IPTV (Internet Protocol Television) [10] is a system where a digital television service is delivered using Internet Protocol over a network infrastructure, which may include delivery by a broadband connection. A general definition of IPTV is television content that, instead of being delivered through traditional broadcast and cable formats, is received by the viewer through the technologies used for computer networks.

IPTV is usually delivered over low cost IP Set Top Boxes designed and developed for a very particular application: the demodulation and decoding of $\mathrm{A} / \mathrm{V}$ signals, and their presentation on a TV display.

Regulation work must be done to deal with content delivery method such as: linear and nonlinear. In linear method the operator keeps control over the time to transmit or stream such content. While non-linear method grants subscriber the control to decide when to start a program, similarly as Video-On-Demand (VoD). 
TISPAN's IPTV architecture couples IPTV (Fig. 4) with the core IMS network itself [11].

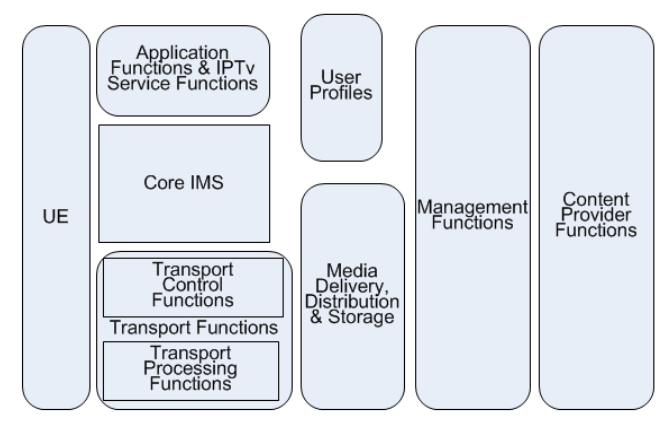

Figure 4. IPTv system architecture

\subsection{ENUM}

Basically, ENUM (TElephone NUmber Mapping) [12] allows mapping an E.164 address to an Internet Domain Name. E.164 addresses are the typical phone numbers used in the PSTN and other networks. Using ENUM, an E.164 address can be translated to N contact forms:

- $\quad$ SIP URI

- H.323 URI

- Fixed number

- Mobile number

- Fax

- E-mail address

- Web page URL

- LDAP server IP address

ENUM [13][14][15][16] was defined in RFC 2916 and updated by RFC 3761. ENUM works like a DNS service using NAPTR ("Naming Authority Pointer") DNS registers. These NAPTR registers are defined in RFC 2915 and updated by RFC 3403. ENUM uses a specific NAPTR: "E2U" (E.164 to URI).

The answer to an ENUM query can be one or more URIs (Fig. 5), being a very important characteristic of ENUM, as it enables many services. Each of them identifies a resource or service associated to the E.164 number. This is the way an ENUM service can forward to N contact forms from an E.164 number. The standard allows defining preferences for each of the contact forms. 


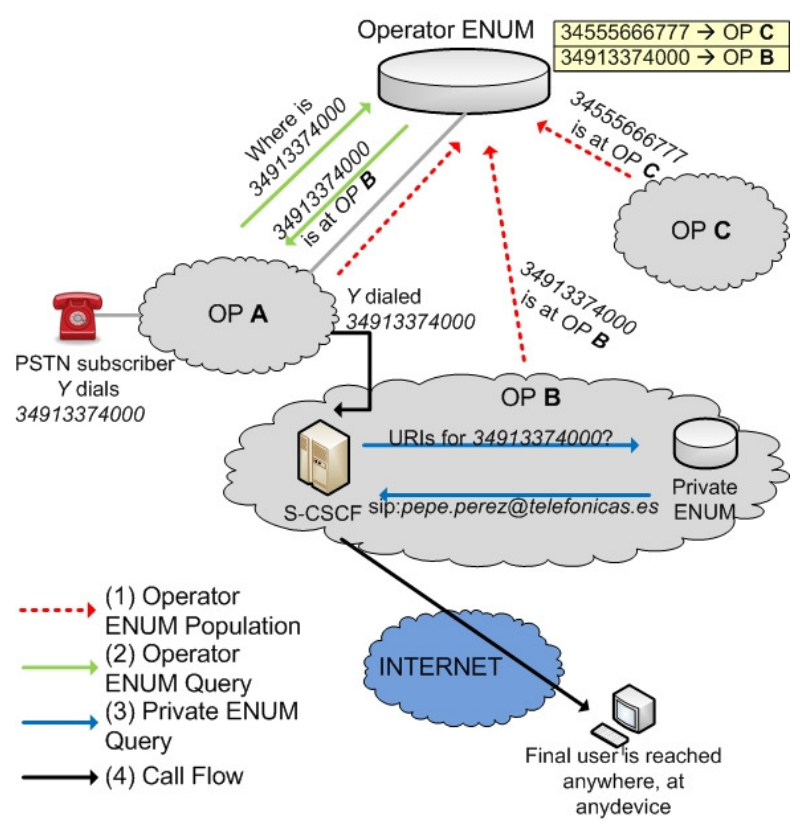

Figure 5. ENUM

\subsection{IMS Client}

The IMS deployment in a fixed-mobile convergent multi-access environment is only possible by extending the usage of IMS to fixed broadband networks such as xDSL, cable TV and IPTV. Nevertheless, many hurdles exist on the way before such vision can be realized, for example: currently an IMS client implemented in a mobile phone will not be able to operate in any wireless LAN environment . It is not only because of the differences between IMS and the standard SIP, but also because of the differences in terms of fundamental subscription differences between the mobile world and the fixed one. Indeed, in the mobile world, each user is associated to one subscription, while in the fixed network a whole household with many users can share one subscription. Furthermore, the authentication schemes used in the WLAN hotspots vary widely and they are different to the ones used in the mobile network. Consequently, due to the lack of compatibility, the IMS services will not work in the WLAN hotspots.

As a result of these fundamental differences, there is no satisfactory mechanism defined for smooth handover between a wireless LAN environment and a 3G network. Besides, current IMS client implementations do not operate in a plain IETF-SIP environment. However, IMS cannot be deployed of in a fixed-mobile convergent multi-access environment unless it is able to operate both with IMS and with SIP.

The success of our testbed results rely on the availability of a client software that emulates IMS signaling as far as possible. 


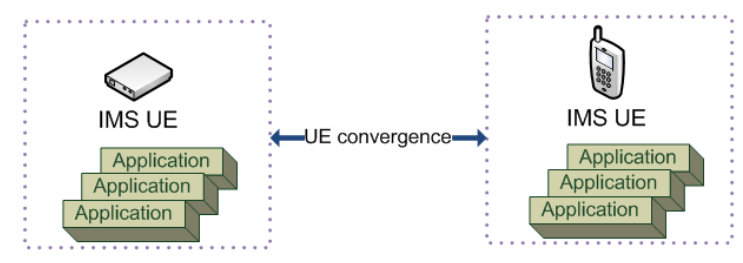

Figure 6. Convergence client

There exists a number of opensource IMS Clients with support on distinct platforms and offering IMS functionality for specific services.

The motivation of the Mobicome project is to expand IMS to cover usage on both mobile and fixed broadband networks, enabling it to be a common service delivery platform on top of fixed, mobile and IP-based networks. This calls for an IMS client framework that is developed for heterogeneous devices, and that can be used for both mobile phones, stationary PCs, set-topboxes and so forth.

As there is often a tight binding and nearly one-to-one mapping between the IMS-based application and a specific underlying service enabler of the existing IMS client frameworks, the utilization of an open-source IMS client was considered adequate for the testing purposes included in the MOBICOME project and to maintain the client implementation located on a terminal, in this case a PC.

\subsection{Related work}

Much work has been done recently regarding the incorporation and implementation of service enablers into IMS architecture in order to solve specific needs of communication. Several fields have already been successfully covered as in medical environments with mHealth [17] where patients are provided with an enhanced privacy protection tool of mHealth presence framework. A sharp implementantion which is focused on dealing with complexity arisen from bringing together legacy networks with IMS is fully described by Telkom Institute of Technology [18] while providing accurate statistical data of performance tests.

Moreover, some work has been focused on the client side with proposals such as Android-based architectures [19] to deal with QoS, security, extendibility and IPTv [20] content delivery issues inherent to existing IMS clients.

The works enlisted above deal only with certain specific needs, therefore, the proof-of-concept architecture presented in this paper is intended to fill the gap existing in the provision of services among distinct service delivery platforms using IMS as a common core with abilities to allow multiple service providers to get interconnected. 


\section{MOBICOME ARCHITECTURE FOR ENUM-3G-IPTV CONVERGENCE}

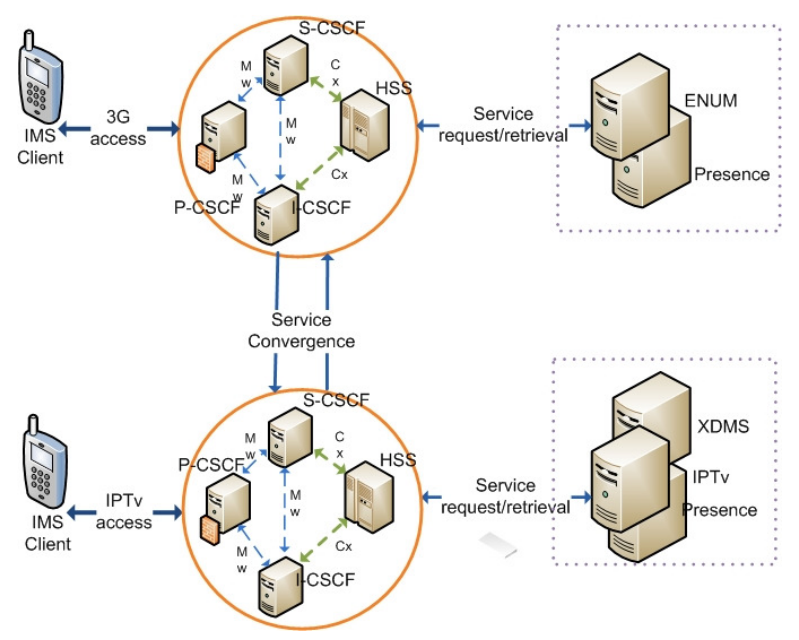

Figure 7. Mobicome overall architecture

The IMS Core acts as the core element of the service delivery platform for the convergence of services. In order to expose the powerful capabilities of the core networks to the end-user applications it is required to define suitable service enabler dedicated to manage the adequate service provision. In the Mobicome project an XDMS service enabler has been integrated in the architecture along with the ENUM and IPTv environment.

In other hand, the end-user equipment should be capable of connecting to both IPTV and 3G access networks and dealing with protocols of the core IMS (in order to support basic capabilities of Operated Telecommunication Networks) and services offered by the enablers (in order to use specific functionalities required by the service).

Both access networks ( $3 \mathrm{G}$ and IPTv) connect to the same IMS Core (but samely can be connected to different cores (say, different Domains are involved). 


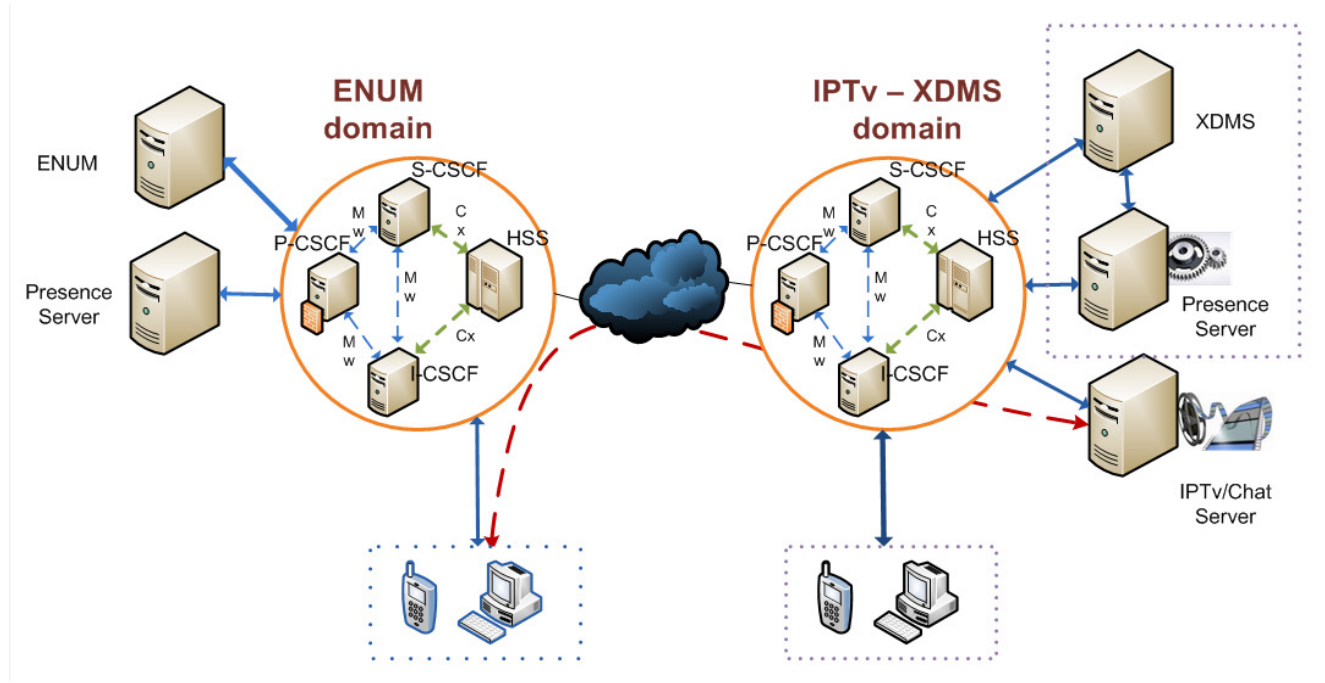

Figure 8. Mobicome detailed architecture

\section{SERVICE CASES}

A variety of IMS service scenarios have been proposed and considered by the MOBICOME partners. These scenarios were focused on the user's perspective regardless of the implementation issues.

This process led to the identification of a number of possible advanced IMS service scenarios. The selection process then condensed the scenarios onto a fewer number of Use Cases that contain only unique characteristics. The selection criteria were: broad domain coverage; attractiveness to the user; technical challenging and interesting; and realistic goal. Through a selection process, seven have been selected:

- Multi-service session and rich communication

- Session transfer and FMC in multi-access

- Secure e-stock trading

- IPTV service device transfer

- Sharing IMS profiles in the same device and IPTV applications

- Family Portal and Web 2.0 applications

- IMS interconnection between Spain and Norway

In this context, several service cases have been identified where SIP messages are routed properly to clarify the service importance of our converged architecture. To exemplify the enhanced functionality provided in the MOBICOME testbed, a working scenario has been selected due to its involvement with every single layer of the architecture. This service case is related to Session transfer and FMC in multi-access with ENUM, ENUM provides a way to translate a standard telephone number to any URI. This means that any user in the PSTN can reach any other user located in any network. This functionality can be improved if not only the translation between the PSTN and the URI are kept in the ENUM database, but also user preferences like white lists, blacklists, presence information, etc.

As an example, Fig. 9 describes the SIP messages exchanged among the communication entitities in order to obtain the physical address of a subscriber. In this scenario, we can highlight the work done on the AS to intercept the ENUM query before arriving the ENUM server itself. This operation guarantees the proper control of the operation and the optimous divertion of the call to 
the adequate IP address in use by the callee. According to the IMS standard, when the S-CSCF receives a SIP request with a TEL-URI as the Request-URI, the S-CSCF tries to translate this URI to a SIP-URI by performing an ENUM lookup.

The architecture had to be modified so that an Application Server does the ENUM lookup instead of the S-CSCF. This is because the translation will not only involve the information retrieved by the ENUM lookup, but other information such as presence status and user call preferences (the most suitable device for the originating session capabilities, white and black lists, the A number, time of the day and day of the week). These operations are performed by the A.S.

The IMS Core (Open IMS Core) has been modified so that it supports standard functionality that was not implemented at the present time. With this modification it is possible that the S-CSCF proxies requests sequentially by taking into account the q-parameter of the Contact header used by the user agents in the registration process. This operation allows creating more interesting scenarios.

The process is as follows (Fig. 9):

- The S-CSCF redirects the INVITE requests it receives to the Application Server (AS).

- The AS checks if the Request-URI is a TEL-URI; if so, it will perform an ENUM lookup to get the possible destination URIs.

- The AS performs a presence lookup for these URIs in order to obtain their presence status.

- The AS performs a lookup to a database to retrieve user information and possibly in addition their call preferences.

- The AS parses all this information in order to get the preferred URI to route the INVITE. It will then modify the Request-URI of the INVITE to the preferred URI and send the INVITE back to the S-CSCF through the ISC interface.

- The call setup continues as defined in the standard.

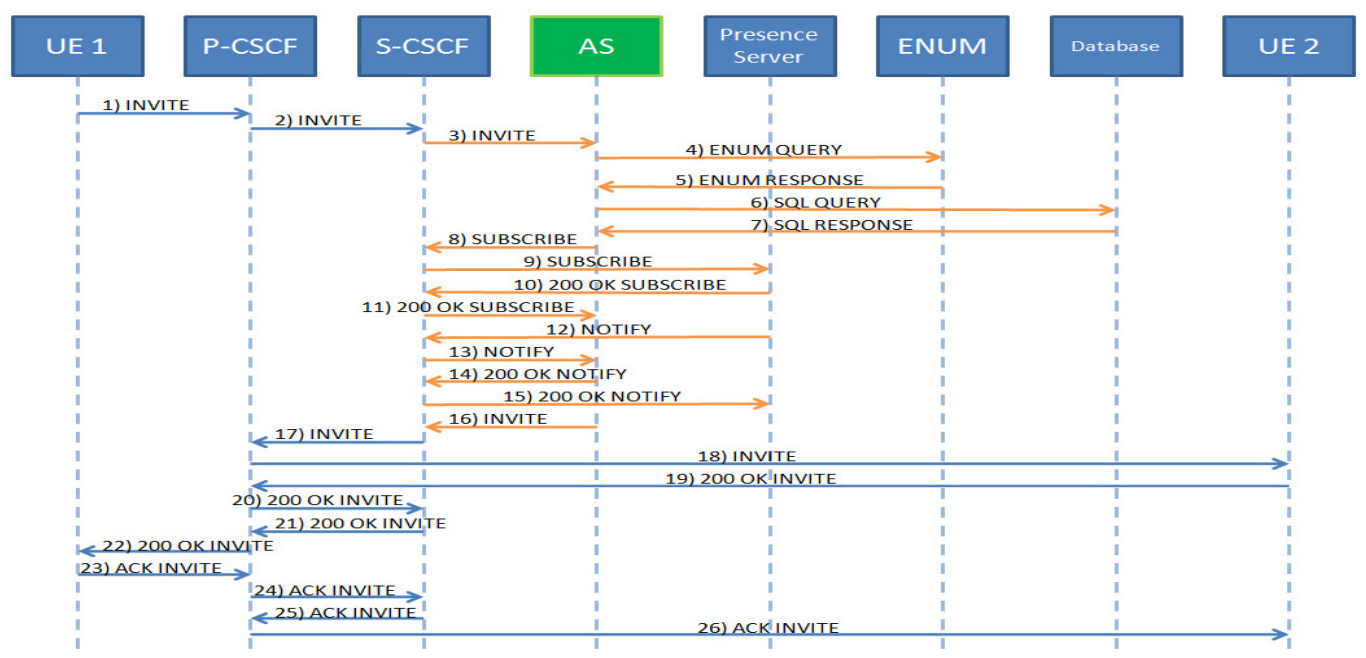

Figure 9. Service case 


\section{IMPLEMENTATION}

\subsection{Proof-of-concept}

In order to demonstrate the results of the project, 3 testbeds have been set up. Two of them gather the results obtained by the Spanish partners and the other one the results from the Norwegian and Swedish sides. All of them demonstrate several advanced services in an intra operator domain. These testbeds were later interconnected to enable an inter operator service deployment.

A set of scenarios that can be provided on top of the testbeds using IPTv environment, XDMS profiles and the enhanced ENUM functionality. The interconnection allows to test a real environment for a multi supplier multinational service test.

Our testbed is based on open source software with active development and it was mostly deployed over Linux-based operating systems such as UBUNTU. The implementation has as basis the Open IMS Core [21] [22] which is an Open Source implementation of IMS Call Session Control Functions (CSCFs) and a lightweight Home Subscriber Server (HSS), which together form the core elements of all IMS/NGN architectures as specified today within 3GPP, 3GPP2, ETSI TISPAN and the PacketCable intiative. The four components are all based upon Open Source software (e.g. the SIP Express Router (SER) or MySQL).

The client side is represented by the UCT IMS Client [23] which is designed to be used in conjunction with the Fraunhofer FOKUS Open IMS Core. The client has been developed by the Communications Research Group at the University of Cape Town, South Africa.

This client supports AKA authentication, and emulates IMS signaling as far as possible. The current version supports voice and video calls, pager-mode and session-based instant messaging, Presence, an IPtv viewer and an XCAP client.

The repository of applications is a Sailfin Application Server [24] which is based on robust and scalable SIP servlets technology on top of a deployment-quality, Java EE-based GlassFish. It provides high- availability and clustering features, while integrating with existing GlassFish services.

OpenXCAP [25] was used in order to handle XML documents as it is needed to manage buddy lists and subscription policies, (like in SIP SIMPLE environments) presence info or other type of events published using SIP protocol.

Presence service is provided via OpenSIPS [26] which in turn acts as a Presence Agent.

The Mobicome project has analyzed, designed, implemented and tested solutions enabling seamless access to the different wireless technologies like UMTS, EDGE, Wireless LAN, besides of providing a unified environment of identification, authentication and authorization of the users and a unified service architecture working as a Uniform Service Delivery Platform (Fig. 10). 


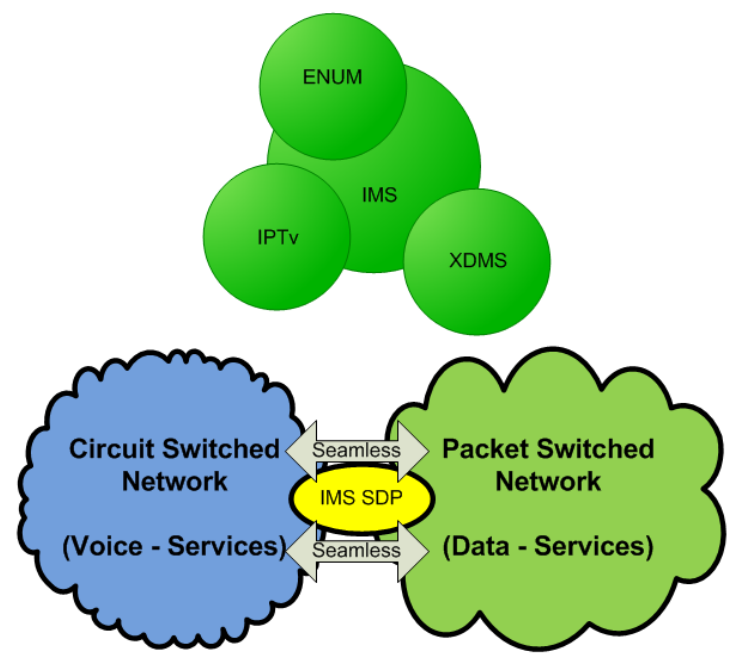

Figure 10. Service Convergence

Our architecture considers an integrated service management model converging IPTv, ENUM, XDMS domains using a common IMS core. These environments may be allocated in different service domains and linked by the I-CSCF of the IMS core.

\subsubsection{XDMS}

Presence information management is considered as a vital element in today's communications systems. PUBLISH, SUBSCRIBE and NOTIFY requests from user equipments must be handled properly by considering presentities' willingness to share information with watchers.

The XDMS is the service in charge of dealing with users' preferences regarding allowing/blocking subscriptions.

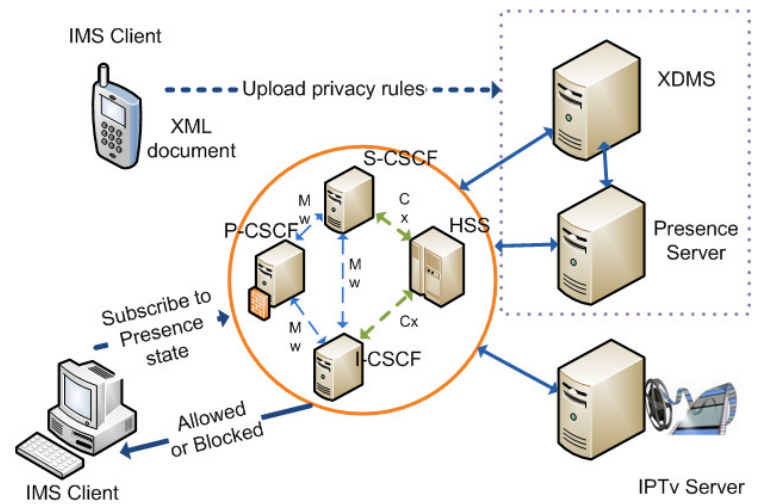

Figure 11. Data communications 


\subsubsection{IPTv}

A generic solution architecture for having an IMS client on a STB would be to have an IMS client presenting a web interface. The STB could then access the IMS client through the Middleware (web browser), being the IMS client installed in a different hardware. There should be noticed that applications in an IPTV STB are always accessed via web browser, even when they are installed on the STB device itself. However, as the scope of the project is not to port the Mobicome IMS client to a particular STB model, this solution is considered more convenient mainly because of its great generality.

The architecture of the solution is presented below in Fig. 12:

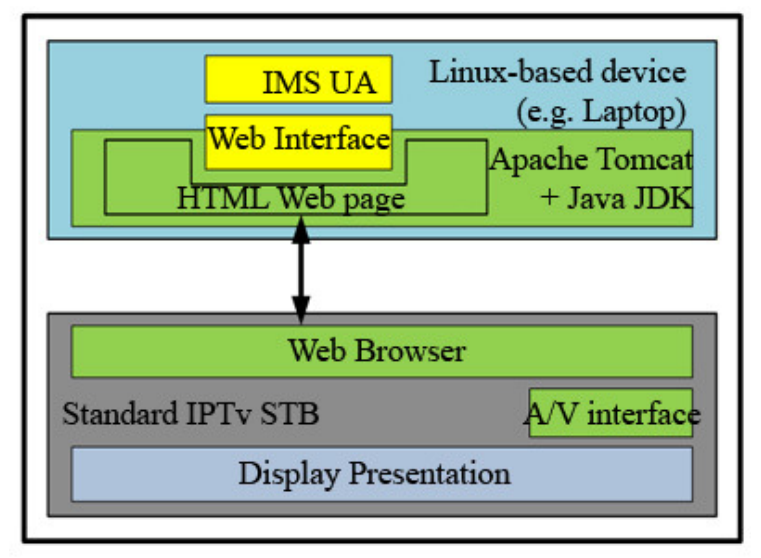

Fig. 12. Mobicome IPTV architecture

A simple chat application has been implemented so as to demonstrate the STB - IMS client functionalities. By means of a basic web interface, the STB user is allowed to access and participate in real-time instant messaging sessions, while an IPTV program is displayed in the background.

As it has been mentioned before, the IMS client itself is installed in an independent equipment, along with an Apache Tomcat web server and the Java JDK. This way, it is possible to adapt the application information and present it in an appropriate format so as it can be accessible to the STB's web browser.

For the implementation of this application, UCT IMS Client has been selected. This client has been designed for being fully compatible with Fraunhofer Institute's Open IMS Core. UCT IMS Client is a versatile free source solution which allows the access of modification of its code to adapt it to the requirements of a concrete application.

Specifically, two modules of UCT IMS Client have been modified for our purposes:

- SIP Event Manager: some changes have been made in this module in order to identify the arrival of a new instant message, translate it into HTML format and present it in the web interface.

- Interface Event Manager: our adapted version of the UCT IMS Client also allows the capture of text introduced by the IPTV service and its presentation it in the web interface. 
The web interface has been implemented following a simple, general approach so as to avoid compatibility problems with browsers of different STBs. Simultaneous presentation of video and instant messaging contents is possible due to the two-layered, configurable transparency structure of STB display. Register and deregister of participants in the IM session are shown in the screen, as well as text messages, in different font formats for the sender and the receiver. Additionally, conversation logs are saved while IM session is active.

As a next step, enabling direct input of text messages by means of the STB's wireless keyboard would improve the usability of the proposed demonstrator solution. However, some problems must be faced in order to make this possible, such as compatibility with the different web browsers, or those related to security and access permissions to the machine where UCT IMS Client is installed.

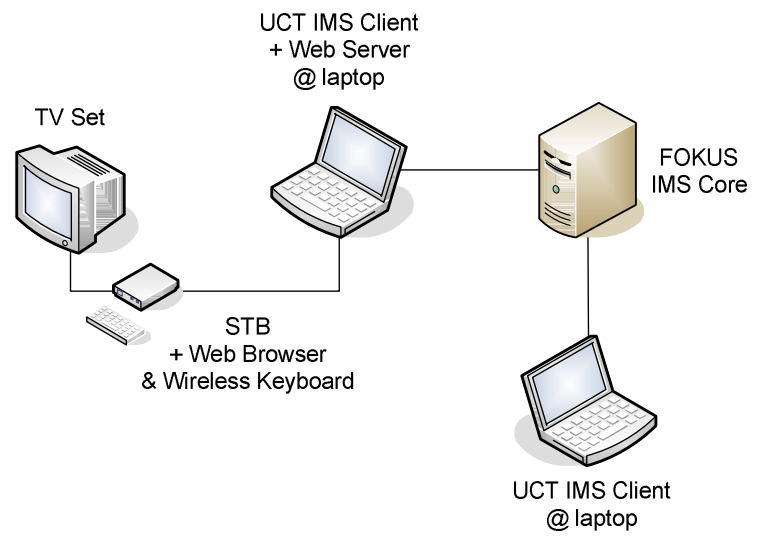

Figure 13. Mobicome IPTv environment

\subsubsection{ENUM}

The Mobicome architecture shows how ENUM can be integrated with IMS to provide improved user subscription management. ENUM provides a way to translate a standard telephone number to any URI. This means that any user in the PSTN can reach any other user located in any network. This functionality can be improved if not only the translation between the PSTN and the URI are kept in the ENUM database, but also user preferences like white lists, blacklists, presence information, etc. 


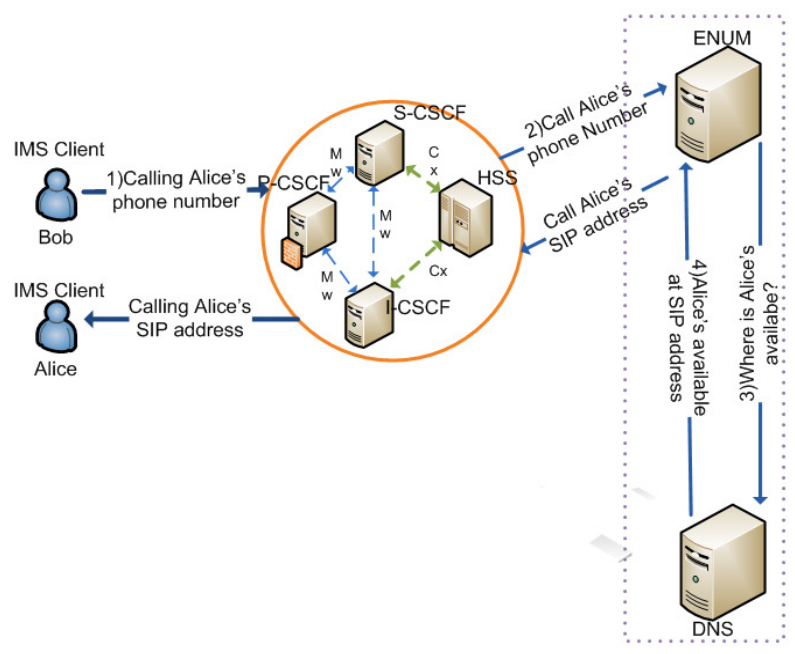

Figure 14. Mobicome ENUM environment

All the components focus on the most popular telecommunication service, namely voice communication or telephony but most importantly show the possibility of combining IMS with state-of-the art Web 2.0 technologies to offer useful and user friendly services to the users.

\subsection{Deployment}

The end user can be provided with different services thanks to the convergence of the fixed and mobile networks and the appropriate technology like the use of the IMS platform.

There is also a need for testing these services when they are not locally to the end users, this means within the same region or country, and moreover protocol interfaces have to deal with different servers and applications.

The first need appears when a user requests for mobility and roaming, so the authentication and authorization queries are bound for his origin country. Nevertheless not only these protocols can have some delay because of the geographical distance, but the applications themselves could be spoilt if timers are time-out or excessive jitter appears at the startup of the signaling process or during the applications reproduction.

Two issues have to be taken into account, transport or connectivity of the applications to the end users when they are not in the region and second, the quality to be given to each of the services involved in the tests. This implies to supply enough bandwidth capacity and/or at the same time to bear in mind but maybe this situation is not always possible so some kind of marking, prioritize and queuing would be a good solution for the low bandwidth environments.

The operating testbed has been deployed over 3 physical premises: Technical University of Madrid (UPM), Telefonica I+D (both in Spain) and Telenor Oslo in Norway. 


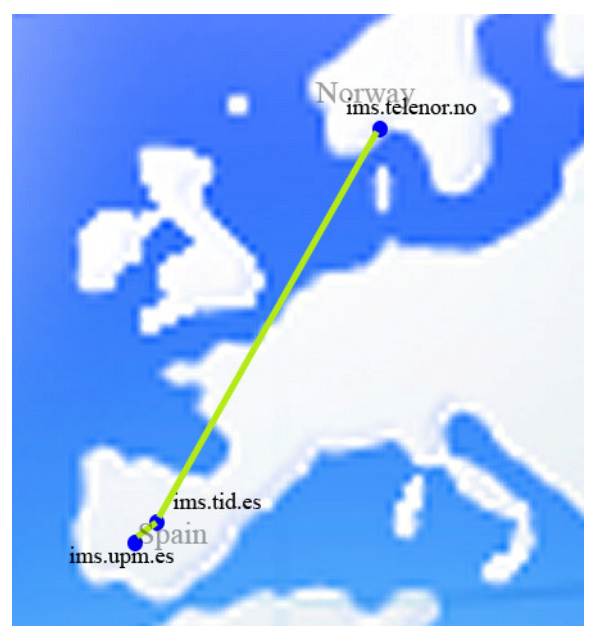

Figure 15. Territorial deployment

The interconnections of these 3 testbed is done via Virtual Private Networks (VPNs). For the international interconnection (Spain-Norway) and due to quality-of-service requirements, the backbone of the network is provided by an international carrier, Telefonica Wholesale, who took the responsability of implementing, configuring and management of the whole solution end to end by means of a VPN with quality of service, marking in the origin of the traffic, in both CEs and remapping and queuing in the trunk networks.

\section{CONCLUSION}

In order to obtain/provide an optimal service in future internet environments, current management architectures shall be able to integrate seamlessly with upcoming service architectures and ease the provision of new complex services that demand converging environments.

Based on the experience gained during MobiCome we can conclude that IMS Core is fully capable of supporting service convergence if it is complemented with a suitable set of enablers. The flexibility of IMS architecture and functionalities, complemented with the facilities by the enablers as defined by OMA, creates the Service Deliverable Platform for such service converge scenarios. In this area it is still required to work on service creation methodologies and tools, because service has to be manually configured and deployed over the convergent scenario.

But on the other Side, a problem not currently afforded is the client side of the services and applications. Standards of International body say mostly nothing about clients, so it is not possible to find a single client compliant with overall IMS capabilities, and we have to adapt some of them to the network access, and end-user terminal. In order to achieve a real fixedmobile convergent multi-access environment much work shall be done in the client side to solve seamless access to services by the end users. Otherwise proprietary solutions will dominate the near future.

This paper has explained the testbed developed in the MOBICOME project which among its goals included the definition of an architecture and deployment to manage the content of IPTv, ENUM and IMS environments running with a common IMS core. 


\section{ACKNOWLEDGEMENTS}

The authors would like to thank the contributions from all the partners of the MOBICOME project. This work is part of the MOBICOME project whose activities are carried out within the scope of the EUREKA program funding. The MOBICOME Consortium includes: Telenor, Linus, Oslo University College, Ericsson Spain - HyC Soluciones de video y Comunicaciones, Universidad Politecnica de Madrid, TIWS, Telefonica Investigacion y Desarrollo S.A, HuaWei, Blekinge Institute of Technology, Ubisafe, and HiQ.

\section{REFERENCES}

[1] [IMS 04]Georgios Tselentis, John Domingue, Alex Galis, Anastasius Gavras, David Hausheer, Srdjan Krco, Volkmar Lotz, Theodore Zahariadis, Towards the Future Internet - A European Research Perspective, 2009, ISBN 978-1-60750-007-0

[2] [IMS 06]The MOBICOME Project, 2009, http://www.mobicome.org.

[3] [3GPP 01] 3GPP TS 23.228 V8.8.0 (2009-03), Technical Specification, 3rd Generation Partnership Project; Technical Specification Group Services and System Aspects; IP Multimedia Subsystem (IMS); Stage 2 (Release 8).

[4] [IMS 03] Poikselka M., Niemi A., Khartabil H., Mayer G., "The IMS: IP Multimedia Concepts and Services", John Wiley \& Sons, 2006, ISBN 0-470-01906-9

[5] [3GPP 02] 3GPP TS 23.292 V9.2.0 (2009-06), Technical Specification 3rd Generation Partnership Project; Technical Specification Group Services and System Aspects; IP Multimedia Subsystem (IMS) centralized services; Stage 2 (Release 9)

[6] [IMS 02] Camarillo G., García-Martín M., "The 3G IP Multimedia Subsystem (IMS): Merging the Internet and the Cellular Worlds", John Wiley \& Sons, 2006, ISBN 0-470-01818-6.

[7] [OMA 01]OMA, Open Mobile Alliance, http://www.openmobilealliance.com/.

[8] [OMA 02]OMA, XML Document Management Architecture, Approved Version 1.1 - 27 Jun 2008.

[9] [IETF 05] IETF J. Rosenberg, "RFC 4825 - The Extensible Markup Language Configuration Access Protocol," May 2007.

[10] [ITU 01] IPTv Focus Group Proceedings 2008, http://www.itu.int/publ/T-PROC-IPTVFG2008/en

[11] [ETSI 01]ETSI RTS 182027 V2.2.0 (2008-11).

[12] [ITU 02] ENUM, http://www.itu.int/osg/spu/enum/, 2009

[13] [IETF 01] M. Mealling, R. Daniel, IETF, RFC 2915 - The Naming Authority Pointer (NAPTR) DNS Resource Record, September 2000, http://tools.ietf.org/html/rfc2915

[14] [IETF 02] P. Faltstrom, M. Mealling, IETF, RFC 3761 - The E.164 to Uniform Resource Identifiers (URI) Dynamic Delegation Discovery System (DDDS) Application (ENUM), April 2004, http://tools.ietf.org/html/rfc3761

[15] [IETF 03] R. Daniel, M. Mealling, IETF, RFC 2168 - Resolution of Uniform Resource Identifiers using the Domain Name System, June 1997, http://tools.ietf.org/html/rfc2168

[16] [IETF 04] IETF Telephone Number Mapping Working Group, http://www.ietf.org/dyn/wg/charter/enum-charter.html, 2009.

[17] Xin Huang, Yang Jiang, Zuguang Liu, Theo Kanter and Tingting Zhang, "Privacy for mHealth presence", International Journal of Next-Generation Networks (IJNGN) Vol.2, No.4, December 2010 . 
[18] Rendy Munadi, Effan Najwaini, Asep Mulyana, R. Rumani, "Design and implementation VoIP service on Open IMS and Asterisk servers interconnected through ENUM server", International Journal of Next-Generation Networks (IJNGN) Vol.2, No.2, June 2010.

[19] Suman Kumar S.P and Vijay Anand, "A novel scalable architecture on Android to cater end-2-end IMS services to audio video terminals", International Journal of Next-Generation Networks (IJNGN) Vol.2, No.3, September 2010.

[20] Suman Kumar S.P and Vijay Anand, "A robust client architecture on Android to cater end-2-end real-time content management and personalized IPTV services to mobile internet devices", International Journal of Next-Generation Networks (IJNGN) Vol.2, No.3, September 2010.

[21] [IMS 05]P. Weik, D. Vingarzen, and T. Magedanz, "The FOKUS Open IMS Core - A Global Reference Implementation," IMS Handbook - Concepts, Technologies and Services - Mohamed Ilyas and Syad Ahson, pp. 113132, November 2008.

[22] [SW 01] Open IMS Core, Open Source implementation of Open IMS Core, http://www.openimscore.org/.

[23] [SW 02] University of Cape Town, "UCT IMS Client," http://uctimsclient.berlios.de/.

[24] [SW 05] Sailfin Project, https://sailfin.dev.java.net/.

[25] [SW 03] M. Amarascu, "OpenXCAP," Free XCAP server for SIP SIMPLE, http://WWltv.openxcap.org/.

[26] [SW 04] Open SIP Server, http://www.opensips.org/. 